\title{
Informatikunterricht in Deutschland - eine Übersicht
}

\author{
Richard Schwarz ${ }^{1}$ Lutz Hellmig' · Steffen Friedrich²
}

Angenommen: 3. März 2021 / Online publiziert: 6. April 2021

(c) Der/die Autor(en) 2021

\section{Zusammenfassung}

Nunmehr 10 Jahre nach der letzten Synopse zur Situation des Informatikunterrichts in Deutschland wird mit dieser Untersuchung ein aktuelles Bild der informatischen Bildung in den 16 Bundesländern gezeichnet. Dem allgemeinbildenden Charakter der informatischen Bildung entsprechend liegt der Fokus auf Angeboten für einen verbindlichen - durch die Existenz eines curricularen Rahmens abgesicherten - Informatikunterrichts für alle Schülerinnen und Schüler in allen Schularten weiterführender Schulen. Die Grundlage für die Erhebungen bildeten sowohl umfangreiche Analysen bildungspolitischer Dokumente, Stundentafeln und curricularer Vorgaben als auch eine Verifikation der erhobenen Daten durch Expertinnen und Experten in den jeweiligen Bundesländern. Neben einer vergleichbaren Darstellung für jedes Bundesland machen die Übersichten zum Sekundarbereich I und II über alle Bundesländer deutlich, welche Defizite in der Grundlagenbildung zur Informatik noch immer vorhanden sind und das Vorankommen in der Digitalisierung in Schulen zusätzlich hemmen.

\section{Bedeutung des Unterrichtsfachs Informatik}

Im Zeitalter der Digitalisierung verändert sich auch der Alltag in Schulen. Unterricht unterliegt einem Wandel und erfordert durch den Einsatz moderner digitaler Medien andere Rahmenbedingungen. Für viele Berufe ist der tägliche Umgang mit modernen Technologien bereits im eher geschlossenen Umfeld in den Schulen eine gute Orientierung. Diese Veränderungen sind Ausdruck eines Umbruchs in den Leitmedien, den wir schleichend bereits im Alltag erleben. Hier muss die Schule für die Allgemeinbildung Antworten finden. Und diese sind eben nicht nur auf das Vorhandensein digitaler Medien, deren Beurteilung und den Austausch von Materialien über verschiedene Plattformen beschränkt.

Aktuell werden verstärkt digitale Arbeitsweisen und damit verbundene IT-Kenntnisse gefordert, es wird vor einer

\begin{tabular}{ll}
\hline CC BY-NC-SA 3.0 DE. \\
\hline $\begin{array}{l}\text { Richard Schwarz } \\
\text { richard.schwarz@ posteo.de }\end{array}$ \\
Lutz Hellmig \\
lutz.hellmig@ uni-rostock.de \\
$\quad \begin{array}{l}\text { Steffen Friedrich } \\
\text { steffen.friedrich@tu-dresden.de }\end{array}$ \\
$1 \quad$ Universität Rostock, Rostock, Deutschland \\
$2 \quad$ TU Dresden, Dresden, Deutschland
\end{tabular}

digitalen Spaltung der Gesellschaft gewarnt und in sehr unterschiedlichen Facetten über Sinn und Unsinn von Bildung im Kontext von digitalen Medien und Informatik diskutiert. Spätestens seit der Veröffentlichung der ersten Ergebnisse vergleichender empirischer Studien wie TIMSS, PISA oder ICILS haben verschiedene Debatten und fast unzählige wissenschaftliche und journalistische Schriften dafür gesorgt, Interesse an einer sogenannten ,digitalen Bildung“ zu wecken, ohne deren Gegenstand genauer zu beschreiben und damit wirkliche Antworten auf Fragen zu digitalen Phänomenen zu ermöglichen [1].

Die Fähigkeit zum bloßen Hantieren mit Arbeitsgeräten und Werkzeugen ist genauso wenig Zeichen einer Kompetenz im künstlerischen oder naturwissenschaftlichen Bereich, wie das Vermögen, Texte auf einer Schreibmaschine zu tippen, Zeichen eines kompetenten Sprachgebrauchs ist. Diese Metapher darf konsequenterweise auch auf die ,digitale Bildung" angewendet werden.

Auch hinsichtlich der informatischen Bildung ist nicht die Bedienung einer Anwendung oder eines Gerätes entscheidend, sondern es sind Konzepte zu hinterfragen, um Alltagsphänomene (oder schlicht: Erlebnisse bzw. notwendige Handlungen) zu verstehen und ihnen gestaltend begegnen zu können. Informatische Bildung und Nutzung digitaler Medien im Sinne einer Medienbildung befinden sich im Schulalltag in einem ständigen Begründungszwang, obwohl in Wirtschaft und Gesellschaft viele Anwendungen aus diesen Bereichen längst zur Normalität gehören. Gleichzeitig 
werden die öffentlichen Medien wie Presse und Fernsehen nicht müde, von Problemen in der Nutzung moderner Informationskanäle durch Kinder und Jugendliche zu berichten. Vielfältige Formen der Bildung in diesem Bereich wurden und werden gefordert. Computerführerschein, Medienpass und viele andere Wortschöpfungen reihen sich aneinander. Die Herausforderungen an die Bildung betreffen insbesondere die Interaktivität, die Kreativität oder auch das Gestalten. Dabei geht es nicht darum, ,etwas mit digitalen Medien“ zu machen, sondern vielmehr darum, digitale Anwendungen selbst zu entwickeln und kreativ zu verändern. Dafür sind dann auch Modellieren und Realisieren (eigentlich Implementieren) erforderlich, Kernkompetenzen einer informatischen Bildung. Es erscheint in diesem Kontext überfällig, eine systematische Bildung - die Informatik und die digitalen Medien betreffend - einzufordern.

Es geht nicht um ein „Mehr an Informatikunterricht“, sondern um die Anerkennung für ein allgemeinbildendes Schulfach Informatik für alle Schülerinnen und Schüler überhaupt. Die bildungspolitischen Debatten um die Einführung von Fächern und die vorhandenen vollen Stundentafeln in allen Schularten sind eine Seite. Die andere Seite ist die aktuelle Notwendigkeit, essenzielle Grundlagen der Veränderungen in Wirtschaft und Gesellschaft, insbesondere die Ausprägung der dafür notwendigen Kompetenzen, didaktisch geeignet in der Allgemeinbildung für alle fest zu verankern.

Schüler und Schülerinnen brauchen kein Spezialwissen, aber ein grundsätzliches Verständnis für Fragen der maschinellen Verarbeitung von Informationen und Daten. Sie sollten nicht nur eine ,passive Konsumhaltung" einnehmen, sondern beurteilen können, welche medizinischen, ökologischen und ethischen Folgen die Digitalisierung oder die künstliche Intelligenz haben. Sie sollten auch einschätzen können, welche Grenzen die Digitalisierung hat [2].

Da informatische Bildung sich nicht in der Bedienung einer Anwendung oder eines Gerätes erschöpft, sondern genau die notwendigen Informatikkonzepte hinterfragt, um Erlebnisse bzw. notwendige Handlungen mit digitalen Anwendungen - also Informatiksystemen - zu verstehen, muss sie im Kontext der Bildung in einer digitalen Welt unbedingt verortet werden.

Es gibt in den Bundesländern große Unterschiede in der Einordnung eines Schulfaches Informatik in die jeweilige Stundentafel. Das betrifft neben den Sekundarbereich I in den weiterführenden Schulen vor allem Informatikkurse in der Oberstufe auf unterschiedlichem Niveau. Das Interesse und die in der Schule bereits vorliegenden Angebote sind in verschiedenen Studien der letzten Jahre bereits sichtbar geworden.
Zur Verbesserung der informatischen Bildung sind Maßnahmen zur Realisierung eines durchgängigen Informatik-Unterrichts umzusetzen [3].

Ein Fach Informatik als Kern der Ausprägung digitaler Kompetenzen dient der Darstellung und Systematisierung von Begriffen und Grundzusammenhängen der Informatik sowie der Vervollständigung von Kenntnissen und Einsichten zu grundlegendem Allgemeinwissen. Der Informatikunterricht stellt (ähnlich dem Mathematikunterricht im Rahmen einer mathematischen Bildung) ein wichtiges systematisierendes Element dar. In den durch die Gesellschaft für Informatik e.V. publizierten ,Grundsätzen und Standards für die Informatik in der Schule“ sowie den „Kompetenzen für informatische Bildung im Primarbereich“ [4-6] sind Kompetenzen dargestellt und erläutert, die Anforderungen an eine informatische Bildung beschreiben, Antworten zu Alltagsphänomenen ermöglichen und an Beispielen zeigen, was Informatikbildung erreichen soll. Die Debatte um Schulfächer ist in diesem Zusammenhang ein zeitloses Thema:

Die Schulfächer sind eine künstliche Konstruktion, kommen im Leben selbst nicht vor, was vielfach als Beweis für die Lebensfremdheit der Schule gilt. Sie sind die einzige Möglichkeit, die komplexe Wirklichkeit des Lebens denkend zu ordnen. ... Bei der Festlegung eines Kanons muß schließlich auch bedacht werden, daß jedes Schulfach sich hinsichtlich seiner Themen und Methoden deutlich von den anderen unterscheiden muß, damit es sich den Schülern als ein in sich vernünftig bearbeitbarer Aspekt der Wirklichkeit darstellen kann [7, S. 24, 26].

Nur ein Schulfach Informatik kann in diesem Sinne die notwendigen Sach-, Methoden- und Handlungskompetenzen ausbilden und Defizite im vorhandenen Fächerkanon beseitigen. Letztlich ist informatische Bildung allerdings nur in einem Gesamtkonzept zu verwirklichen, das Beiträge zur Medienerziehung ebenso einschließt wie den Unterricht in einem eigenständigen Fach. Gegen ein Schulfach Informatik für alle spricht sachlich nichts. In der Argumentation wird oft angeführt, dass Programmierkenntnisse nicht für alle nötig seien. Diese eingeschränkte Sicht auf die Gegenstände des Faches ist dabei häufig durch die eigene Schulerfahrung entstanden. Informatik in der Schule ist einfach mehr.

Die benutzten Anwendungen und Programmiersprachen sind immer Werkzeuge zur Vermittlung von Inhalten der Informatik, zum Erlernen der Arbeitsmethodik des Faches und zum Beurteilen des Einsatzes der jeweiligen Systeme. So sind beispielsweise die fundierte Einführung in Standardsoftware, Strukturierung von Informationen und Abläufen, Suchstrategien im Internet, Aufbau und Funktions- 
weise des Internets oder auch die Beschreibung einfacher, automatisierbarer Vorgänge ein Beleg für die positive Wirkung der Informatik im Kanon der Unterrichtsfächer. Insbesondere geht es nicht nur um das Produzieren mit digitalen Werkzeugen, sondern auch um die digitalen Produkte selbst. Schließlich werden Modellierungstechniken benötigt, um zu verschiedenen Themen ein vertieftes Verständnis zu erzeugen.

In Deutschland existiert das Schulfach Informatik in der Oberstufe der Gymnasien seit ca. 40 Jahren und hat aus sehr unterschiedlichen Gründen bisher keine bundesweite Anerkennung gefunden. Verschiedene Versuche, die Gegenstände eines Faches Informatik integrativ in anderen Fächern oder Profilen einzubinden, können inzwischen bundesweit als gescheitert erklärt werden. Weder die Grundbildung in den 1980er-Jahren noch eine starke gesellschaftswissenschaftliche Ausrichtung Anfang der 1990er-Jahre konnten die Bildung zur Informatik in den Schulen stabilisieren. Die Chance, begleitend zum umfangreich geförderten Projekt „Schulen ans Netz“ die dazu gehörende Informatik-Bildung verpflichtend zu etablieren, wurde Mitte der 1990er-Jahre vertan. In der Vorstudie zur Bildungsinitiative ,Schulen ans Netz" wurde festgestellt:

Fünftes Ziel: Sich-Aneignen von Hintergrundwissen Sich-Aneignen von Hintergrundwissen zum kompetenten und verantwortungsbewussten Umgang mit Netzen, d.h. das Erlangen bzw. Ergänzen einer informatischen Bildung [8, S. 25].

Betrachtet man den Zeitraum und eine Reihe gerade in jüngster Vergangenheit veröffentlichter Vergleichsstudien oder die internationalen Aktivitäten in diesem Bereich, wird erst richtig deutlich, welches Potenzial in den letzten 25 Jahren in Deutschland verschenkt wurde. Erst mit technischen und gesellschaftlichen Veränderungen Anfang der 2000er-Jahre wurde die Debatte bildungspolitisch wieder relevant, ohne bis heute einen Durchbruch zu einer verpflichtenden Informatikbildung zu erreichen.

Der aktuelle Stand zum Informatikunterricht ist in den Bundesländern sehr unterschiedlich und Vergleiche werden seitens der KMK nicht angestellt. Vorliegende Studien aus dem Jahr 2010 [9] sind wegen der positiven Veränderungen in jüngster Zeit nicht seriös zu verwenden. Es zeigt sich, dass man im Sekundarbereich in Mecklenburg-Vorpommern (seit 1998), Sachsen (seit 1992) und Bayern (seit 2001) ein Pflichtangebot in den einzelnen Jahrgangsstufen findet, in den beiden erstgenannten Ländern in allen Schulformen mit meist einer Wochenstunde. Mit großer Anstrengung aus den Schulen heraus hat sich die Situation in Baden-Württemberg (Pflicht in Klasse 7, Wahlpflichtangebot) etwas verbessert.

Das waren Gründe, um erneut eine Studie zum Stand des Informatikunterrichts in Deutschland an weiterführen- den Schulen anzufertigen. Die Untersuchung erfolgte für die Sekundarstufe I (unter Einbeziehung der Orientierungsstufe ab Jahrgangsstufe 5) und für die Sekundarstufe II.

\section{Untersuchungsdesign}

Die Datengewinnung erfolgte durch eine umfangreiche, durch eine Expertenbefragung verifizierte Dokumentenanalyse.

Zunächst nahm die Sichtung curricularer Dokumente auf Inhalte mit Informatikbezug in den Bundesländern einen breiten Raum ein. Ein Curriculum trägt wesentlich zur Bestimmung des Stellenwerts eines Fachs bei. Durch ihren für Lehrkräfte bindenden Charakter haben curriculare Vorgaben in gewissem Maße Einfluss auf unterrichtliche Planungsprozesse und erlauben Rückschlüsse auf die Bildungspraxis [10, S. 12]. Konkret ergaben sich folgende Fragen.

- In welcher Form ist das dem curricularen Dokument zugrundeliegende Fach organisiert?

- Für welche Schulart bzw. welchen Schulbereich gilt das Dokument?

- Für welche Jahrgangsstufen gilt das Dokument?

Schon aus den curricularen Vorgaben ergaben sich Hinweise auf die Exklusivität und die Systematik der Vermittlung informatischer Bildung, die in die Kategorien „Eigenständiges Fach“, „Interdisziplinäres Fach“ sowie „Fächerübergreifender Unterricht“ gefasst werden können. Nur wenn Informatikunterricht im Rahmen eines eigenständigen Fachs stattfindet oder der Anteil informatischer Inhalte im Rahmen eines interdisziplinären Fachs explizit ausgewiesen ist, ist eine objektive Vergleichbarkeit der Stundenkontingente gegeben.

Vor diesem Hintergrund wurden für alle Bundesländer Recherchen zu bildungsbezogenen Dokumenten vorgenommen, die auf Gesetzes- oder Verordnungsebene die Verbindlichkeit, die Einbettung und den Umfang informatischer Bildung in den Schularten der Länder regeln.

Bei der Analyse der informatischen Bildung in der Sekundarstufe I lag besonderes Augenmerk auf verbindlichem Informatikunterricht für alle Schülerinnen und Schüler, unabhängig vom Bildungsgang. Im Einzelnen wurden in der Sekundarstufe I folgende Fragestellungen untersucht:

- Mit welcher Verbindlichkeit findet Informatikunterricht statt?

- In welchen Jahrgangsstufen findet Informatikunterricht statt?

- In welchem Stundenumfang findet Informatikunterricht statt? 
Informatikunterricht in Deutschland (Sekundarstufe I)

Stand: August 2020

\begin{tabular}{|c|c|c|c|c|c|c|c|c|c|}
\hline Bundesland & Schulform $\rightarrow$ Abschluss & 5 & 6 & 7 & 8 & 9 & 10 & Prüfung & Hinweise und Anmerkungen \\
\hline \multirow{3}{*}{$\begin{array}{c}\text { Baden- } \\
\text { Württemberg }\end{array}$} & \begin{tabular}{|l|} 
mittlere Reife \\
\end{tabular} & $\times$ & $\times$ & 1 & 1 & 1 & 1 & & - \\
\hline & mittlere und Hochschulreife & $\times$ & $\times$ & 1 & 1 & 1 & 1 & & 1) \\
\hline & Hochschulreife & $\times$ & $x$ & 1 & 2 & 1 & 1 & & 2) \\
\hline \multirow{2}{*}{ Bayern } & mittlere Reife & 1 & 1 & 1 & 1 & 1 & 1 & & 3) \\
\hline & Hochschulreife & $\times$ & 1 & 1 & $x$ & 2 & 2 & & 4) \\
\hline \multirow{3}{*}{$\begin{array}{l}\text { Berlin } \\
\text { y.ay }\end{array}$} & mittlere Reife & $x$ & $\times$ & 1 & $\times$ & 2 & 2 & & \multirow{3}{*}{ 5) } \\
\hline & mittlere und Hochschulreife & $x$ & $\times$ & 1 & $\times$ & 2 & 2 & & \\
\hline & Hochschulreife & $\times$ & $\times$ & 1 & $\times$ & 2 & 2 & & \\
\hline \multirow{3}{*}{ Brandenburg } & mittlere Reife & $x$ & $\times$ & & & & & & \multirow{2}{*}{ 6) } \\
\hline & mittlere und Hochschulreife & $x$ & $\times$ & & & & & & \\
\hline & Hochschulreife & $\times$ & $\times$ & & & 1 & 2 & & 7) \\
\hline \multirow{3}{*}{ Bremen } & mittlere Reife & $\times$ & $\times$ & $\times$ & $x$ & $x$ & $x$ & & \multirow{3}{*}{ 8) } \\
\hline & mittlere und Hochschulreife & $x$ & $x$ & $x$ & $x$ & $x$ & $\times$ & & \\
\hline & Hochschulreife & $\times$ & $\times$ & $\times$ & $\times$ & $\times$ & $\times$ & & \\
\hline \multirow[t]{3}{*}{ Hamburg } & mittlere Reife & $x$ & $\times$ & & & & & & \multirow{2}{*}{ 9) } \\
\hline & mittlere und Hochschulreife & $\times$ & $\times$ & & & & & & \\
\hline & Hochschulreife & $\times$ & $\times$ & & & & & & 10) \\
\hline \multirow[t]{3}{*}{ Hessen } & mittlere Reife & $x$ & $x$ & $x$ & $x$ & $x$ & $x$ & & \multirow{3}{*}{ 8) } \\
\hline & mittlere und Hochschulreife & $x$ & $\times$ & $\times$ & $\times$ & $x$ & $\times$ & & \\
\hline & Hochschulreife & $\times$ & $\times$ & $\times$ & $\times$ & $\times$ & $\times$ & & \\
\hline \multirow{3}{*}{$\begin{array}{l}\text { Mecklenburg- } \\
\text { Vorpommern } \\
\text { 재 }\end{array}$} & mittlere Reife & 1 & 1 & 1 & 1 & 1 & 1 & $\checkmark$ & \multirow{3}{*}{ 11) } \\
\hline & mittlere und Hochschulreife & 1 & 1 & 1 & 1 & 1 & 1 & $\checkmark$ & \\
\hline & Hochschulreife & 1 & 1 & 1 & 1 & 1 & 1 & & \\
\hline \multirow[t]{3}{*}{ Niedersachsen } & mittlere Reife & $\times$ & 2 & 2 & 2 & 2 & 2 & & \multirow{2}{*}{ 12) } \\
\hline & mittlere und Hochschulreife & $\times$ & 2 & 2 & 2 & 2 & 2 & & \\
\hline & Hochschulreife & $x$ & 2 & 2 & 2 & 2 & 2 & & 13) \\
\hline \multirow{3}{*}{$\begin{array}{l}\text { Nordrhein- } \\
\text { Westfalen } \\
\end{array}$} & mittlere Reife & $\times$ & $\times$ & & & & & & \multirow{2}{*}{ 14) } \\
\hline & mittlere und Hochschulreife & $x$ & $x$ & & & & & & \\
\hline & Hochschulreife & $x$ & $x$ & $x$ & $x$ & & & & 15) \\
\hline
\end{tabular}

Abb. 1 Übersicht zum Informatikunterricht in Sekundarstufe I (Stand August 2020). Die Zahlen in den Feldern entsprechen den Unterrichtsstunden pro Woche. 1) Gesamtkontingent 8 UStd./Wo. für Profilfach „Informatik, Mathematik, Physik“; Anteil Informatik insgesamt bei 3 UStd./Wo., 2) Analog mit Anteil Informatik bei 4 UStd./Wo., 3) Wahl- oder Wahlpflicht mit 2 UStd./Wo. in 9/10 möglich, 4) Fach „Natur und Technik“ in 6/7 mit verbindlichem Anteil Informatik; „Informatik“ in 9/10 nur an naturwissenschaftlich-technologischem Zweig, 5) In Jahrgangsstufe 7 oder 8 als ITG mit verbindlichen Anteilen; Einordnung in Pflichtbereich möglich; alternativ auch 3 UStd. in 9/10, 6) Schwerpunktunterricht über alle Jahrgangsstufen von 7-10 mit max. 5 UStd. möglich, meist nur in einer Jahrgangsstufe, 7) Schwerpunktunterricht über mehrere Jahrgangsstufen, meist 9/10 mit 1 bzw. 2 UStd./Wo., 8) In allen Schularten kein Informatikunterricht, auch nicht fächerübergreifend mit festen Anteilen, 9) Wenn angeboten, dann meist 2 UStd./Wo. insgesamt über alle Jahrgangsstufen, 10) Wie oben, Umfang nach Profil unterschiedlich, 11) Als Unterrichtsfach „Informatik und Medienbildung“; Wahlpflicht zusätzlich möglich, 12) Schwerpunktangebot auch nur in Jahrgangsstufe 9/10 möglich, 13) Profil mit Schwerpunktfach Informatik möglich, dann 3-4 UStd., 14) Gesamtkontingent von bis zu 14 UStd. über mehrere Jahrgangsstufen ab 2021: Pflichtfach Informatik in den Jahrgangsstufen 5/6, 15) Gesamtkontingent von 6 UStd. über 2 Jahrgangsstufen

In der gymnasialen Oberstufe haben die Bundesländer aufgrund der KMK-Vorgaben einen geringeren Handlungsspielraum als in der Sekundarstufe I. Vor dem Hintergrund der umfangreichen Wahlmöglichkeiten für alle Fächer und der besonderen Bedeutung der Prüfung in der gymnasialen Oberstufe sind die Fragestellungen für die Sekundarstufe II angepasst worden:

- In welcher Hinsicht werden Festlegungen zur Belegung des Fachs Informatik getroffen?
- Welche Bestimmungen zu den Abiturprüfungen gelten für das Fach Informatik?

- Ist das Fach Informatik den naturwissenschaftlichen Fächern gleichgestellt?

In der Sekundarstufe II erfolgte die Einteilung nicht nach Jahrgängen, sondern nach Orientierungsphase und Qualifikationsphase differenziert. Das erlaubt eine bessere Vergleichbarkeit zwischen den Ländern mit G8- und G9-Bildungsgang, führt zwangsläufig aber dazu, dass die Jahrgangsstufe 10 in Ländern mit G8 sowohl in der Übersicht 


\begin{tabular}{|c|c|c|c|c|c|c|c|c|}
\hline \multirow{3}{*}{ Rheinland-Pfalz } & mittlere Reife & $x$ & & & & & & 16) \\
\hline & mittlere und Hochschulreife & $x$ & $x$ & $\times$ & $x$ & 3 & 3 & - \\
\hline & Hochschulreife & $x$ & $x$ & $\times$ & 3 & 3 & $x$ & - \\
\hline \multirow{3}{*}{$\begin{array}{l}\text { Saarland } \\
\text { Sy }\end{array}$} & mittlere Reife & $x$ & $x$ & $x$ & $x$ & $x$ & $x$ & - \\
\hline & mittlere und Hochschulreife & $x$ & $x$ & $\times$ & $x$ & $x$ & $x$ & - \\
\hline & Hochschulreife & $x$ & $x$ & $\times$ & 2 & 2 & 2 & 17) \\
\hline \multirow{2}{*}{ Sachsen } & mittlere Reife & & & 1 & 1 & 1 & 1 & 18) \\
\hline & Hochschulreife & & & 1 & 1 & 1 & 1 & 19) \\
\hline \multirow{3}{*}{ 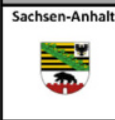 } & mittlere Reife & & & & & & & 20) \\
\hline & mittlere und Hochschulreife & $x$ & $x$ & $\times$ & $x$ & $x$ & $x$ & 21) \\
\hline & Hochschulreife & $x$ & $\mathrm{x}$ & $\times$ & $x$ & 2 & 3 & - \\
\hline \multirow{3}{*}{ 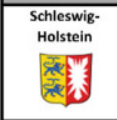 } & mittlere Reife & & & & & & & \multirow{2}{*}{ 22) } \\
\hline & mittlere und Hochschulreife & & & & & & & \\
\hline & Hochschulreife & & & $\times$ & $x$ & 3 & 3 & 23) \\
\hline \multirow{3}{*}{$\begin{array}{l}\text { Thüringen } \\
\text { Fi }\end{array}$} & mittlere Reife & $x$ & $x$ & $\times$ & $\times$ & $x$ & $x$ & 24) \\
\hline & mittlere und Hochschulreife & $x$ & $x$ & . & . & & & 25) \\
\hline & Hochschulreife & $x$ & $x$ & $\times$ & $x$ & & & 26) \\
\hline & \multicolumn{8}{|c|}{ verbindlicher Pflichtunterricht für alle SuS } \\
\hline & \multicolumn{8}{|c|}{ verbindlicher Unterricht nach Wahl durch SuS } \\
\hline & \multicolumn{8}{|l|}{ Wahlunterricht für einige SuS } \\
\hline & \multicolumn{8}{|c|}{ fachübergreifender Unterricht (Anteil } \\
\hline
\end{tabular}

Abb. 1 (Fortsetzung) 16) Gesamtkontingent bis 6 UStd. als schuleigenes Angebot, 17) Gilt für MINT-Zweig (Schulversuch); 2 UStd./Wo. Pflicht in Jahrgangsstufe 8/9 bzw. alternativ 9/10, 18) In Jahrgangsstufe 5/6 „Technik/Computer“ 0,4 UStd./Wo.; [M.I.T-Schulen mit zusätzlich 2 UStd./Wo. ab Jahrgangsstufe 8], 19) In Jahrgangsstufe 5/6 „Technik/Computer“ 0,3 UStd./Wo.; [M.I.T.-Schulen: zusätzlich 3 UStd./Wo. ab Jahrgangsstufe 8], 20) Fach Technik im Profilbereich, Informatikanteile nicht verbindlich, 21) Im Gymnasialzweig analog zum Gymnasium möglich, 22) Gesamtkontingent von bis zu 16 UStd. über 4 Jahrgangsstufen für das Fach „Angewandte Informatik“, 23) Gesamtkontingent von 6 UStd. über 2 Jahrgangsstufen für das Fach „Angewandte Informatik“, 24) Fach Medienkunde über alle Jahrgangsstufen, keine verbindlichen Informatikanteile, 25) Gesamtkontingent von 8 bzw. 9 Ustd./Wo., als Fach „Wirtschaft-Recht-Technik“; Informatik anteilig (unverbindlich), 26) Gesamtkontingent von 6 UStd.

zur Sekundarstufe I als auch in der Übersicht zur Sekundarstufe II aufgeführt wird.

Im Ergebnis der Dokumentenanalyse wurde für jedes Bundesland ein vorläufiges Dossier zur informatischen Bildung angefertigt. Um Fehlinterpretationen zu vermeiden, wurden diese jeweils 2-3 Experten in den Bundesländern zugesandt, die die vorläufigen Dossiers verifizierten, ergänzten und weitere Hinweise zur inhaltlich und formal korrekten Darstellung der Situation gaben. Die verifizierten Dossiers sind bereits veröffentlicht worden [11, 12] und bilden die Grundlage für die aggregierte Darstellung der Situation der informatischen Bildung in Deutschland.

\section{Ergebnisse der Datenanalyse}

Eine Zusammenfassung der Situation in der Sekundarstufe $\mathbf{I}$ ist in Abb. 1 zu finden.

Hieraus ergeben sich folgende Kernaussagen zur Sekundarstufe I

1. In 2 Bundesländern - in Bremen und Hessen - existiert keinerlei Angebot für informatische Bildung im Sekundarbereich I.
2. In 9 Bundesländern wird Informatik ausschließlich nur im Wahlpflicht- und Wahlbereich angeboten.

3. Lediglich in den Bundesländern Baden-Württemberg, Bayern, Mecklenburg-Vorpommern, Saarland und Sachsen gibt es verbindlichen Informatikunterricht in der Sekundarstufe I.

Hier sind jedoch z. T. enorme Unterschiede in der Breite des Angebots bezogen auf die Jahrgänge und betroffenen Schulformen auszumachen (Tab. 1).

4. Mecklenburg-Vorpommern ist seit 2019 das einzige Bundesland, in dem verbindlicher Informatikunterricht für alle Schülerinnen und Schüler durchgängig in den Jahrgangsstufen 5 bis 10 stattfindet.

Die Stundenkontingente für eigenständigen, verbindlichen Informatikunterricht können im Einzelfall eine große Bandbreite aufweisen, sodass verallgemeinernde Aussagen nur mit Einschränkungen zu treffen sind. Während es beispielsweise in Mecklenburg-Vorpommern und Sachsen eigenständigen, verbindlichen Unterricht an allen Schularten im Umfang von einer Wochenstunde gibt, findet dieser im Saarland nur in bestimmten Zweigen am Gymnasium statt; dafür kann dort der Informatikunterricht an Gymnasien im 
Tab. 1 Bundesländer mit verbindlichem Informatikunterricht bezogen auf Zahl der Jahrgangsstufen und Schularten

\begin{tabular}{llll}
\hline & \multicolumn{2}{l}{ Verbindlicher Informatikunterricht in Jahrgangsstufen der Sek I } \\
\cline { 2 - 4 } & In allen & In mehreren & In einer \\
\hline In allen Schularten & $\begin{array}{l}\text { Mecklenburg- } \\
\text { Vorpommern }\end{array}$ & Sachsen & Bayern, Baden- \\
Nicht in allen Schularten, aber mindes- & - & Saarland & - \\
tens in einer & & &
\end{tabular}

Informatikzweig einen Gesamtumfang von 11 Wochenstunden aufweisen.

Ein Überblick zur Situation in der gymnasialen Oberstufe findet sich in Abb. 2.

Aus den Daten lassen sich folgende Aussagen zur Belegung in der Einführungsphase ableiten:

1. In 13 Bundesländern gibt es in der Einführungsphase Angebote für Informatikunterricht im Wahlpflichtbereich, wobei der Umfang zwischen 1 Wochenstunde und 4 Wochenstunden variiert.

2. In 3 Bundesländern gibt es in der Einführungsphase verbindlichen Informatikunterricht: Bayern (2 Wochenstunden), Mecklenburg-Vorpommern und Sachsen (je 1 Wochenstunde).

\section{Zur Belegung in der Qualifikationsphase:}

1. In allen Bundesländern kann Informatik in der Qualifikationsphase auf grundlegendem Anforderungsniveau im Umfang von 2 oder 3 Wochenstunden als Wahlpflichtmöglichkeit belegt werden. Eine Besonderheit stellt Baden-Württemberg dar: Dort ist ausschließlich eine zusätzliche Belegung im Umfang von 2 Wochenstunden möglich.

2. In 12 Bundesländern kann Informatik in der Qualifikationsphase auf erhöhtem Anforderungsniveau im Umfang von 4 oder 5 Wochenstunden angeboten und belegt werden.

In Bayern, Baden-Württemberg, Sachsen und SachsenAnhalt kann Informatik in der Qualifikationsphase nicht auf erhöhtem Anforderungsniveau belegt werden.

3. In Niedersachsen, Sachsen und Thüringen wird Informatik bezüglich der Belegungsverpflichtungen in der Qualifikationsphase gegenüber den naturwissenschaftlichen Fächern gestärkt, da es im Rahmen länderspezifischer Bestimmungen explizit ein naturwissenschaftliches Fach ersetzen kann

Bezüglich der Abiturprüfungen gilt:

1. In allen Bundesländern kann im Fach Informatik eine mündliche Abiturprüfung auf grundlegendem Anforderungsniveau abgelegt werden.

(Mündliche Prüfungen auf erhöhtem Niveau sind lt. KMK-Vorgaben nicht möglich.)
2. Schriftliche Abiturprüfungen auf grundlegendem Niveau sind in 9 Bundesländern möglich.

3. Schriftliche Abiturprüfungen auf erhöhtem Niveau sind in 12 Bundesländern möglich.

4. Andere Prüfungsleistungen, wie z.B. Präsentationsprüfungen oder besondere Lernleistungen, sind in den Bundesländern Berlin, Brandenburg, Hamburg, Hessen, Mecklenburg-Vorpommern, Niedersachsen, RheinlandPfalz, Sachsen und Schleswig-Holstein möglich.

\section{Fazit}

Zum Informatikunterricht in Deutschland ergibt sich aus den Übersichten, vor allem für den Sekundarbereich I, ein sehr differenziertes Bild. Es zeigt deutliche Unterschiede im Hinblick auf die Verbindlichkeit, die jeweilige Schulart und vor allem die Organisationsform eines Fachunterrichts Informatik. Neben einem durchgängigen Pflichtunterricht in wenigen Bundesländern, für den die Inhalte curricular definiert sind, existieren unterschiedlichste Angebote im Wahlpflicht- bzw. im Wahlbereich (nach curricularen Vorgaben oder auch in freier Wahl der Schulen) oder es kommt ein solcher Unterricht überhaupt nicht zustande. Das kann einzelne Schularten, gymnasiale Zweige bzw. Schwerpunkte oder alle Schularten betreffen oder der Informatikunterricht kann in einer, in mehreren oder in allen Jahrgangsstufen stattfinden. Informatische Inhalte können zudem als eigenständiges Fach, im Rahmen eines interdisziplinären Fachs oder in Form fächerübergreifenden Unterrichts vermittelt werden. Diese Auflistung macht die Schwierigkeiten des Vergleichs deutlich, weil die Differenzen in der Struktur der Schullandschaft und in den Verantwortlichkeiten für Inhalt und Umfang zwischen den Bundesländern kaum vergleichbar darstellbar sind. Mit der Kategorisierung von Schulformen und der Art der hier gewählten Darstellung (einschließlich der Hinweise zu speziellen Regelungen) scheint ein gewisser Vergleich möglich, auch wenn dabei von einzelnen Facetten in den Bundesländern abstrahiert werden musste.

Bezugnehmend auf die letzte vergleichende Analyse [9] fällt das Ergebnis insgesamt ernüchternd aus. Die Forderungen von Wirtschaft und Gesellschaft, die die Gesellschaft für Informatik mit der Vorlage von Standards für den Informatikunterricht [4] bereits inhaltlich konkret untersetzt hat, 
Abb. 2 Übersicht zum Informatikunterricht in Sekundarstufe II (Stand August 2020). Die Zahlen in den Feldern entsprechen den Unterrichtsstunden pro Woche. 1) Profilfach „Informatik, Mathematik, Physik" mit 4 UStd./Wo. in der Orientierungsphase; Anteil Informatik ca. 1 UStd./Wo., 2) Gleichstellung in Prüfung nur am Naturwiss-Techn. bzw. WirtschaftsWiss. Gymnasium, 3) Wahlpflichtfach geplant, 4) Orientierungsphase auch 3 UStd./Wo., 5) Orientierungsphase (bei ausreichendem Kontingent); auch 4 UStd./Wo.,

6) Nur als profilgebendes Fach, 7) Orientierungsphase ohne $\mathrm{Zu}$ ordnung von UStd. (Festlegung durch Schule, 2-3 UStd./Wo.); Grundkurs auch mit 3 UStd./Wo. möglich, 8) Orientierungsphase: Fach „Informatik und Medienbildung" in Jahrgangsstufe 10, 9) durchgängig nur im mathematisch-naturwissenschaftlichen Schwerpunkt, 10) Im Informatikzweig dreistündig als verpflichtendes Fach, 11) Ab 2023: M.I.T.-Schulen mit Leistungskurs (5 UStd./Wo.) und schriftlicher Abschlussprüfung, 12) Nur im naturwissenschaftlichen Profil wählbar, 13) Gilt nur für Spezialklassen an math.naturwissenschaftlich-techn. Gymnasien

\begin{tabular}{|c|c|c|c|c|c|c|c|c|c|}
\hline \multirow{3}{*}{\begin{tabular}{|c} 
Bundesland \\
Baden- \\
wirttemberg \\
yty
\end{tabular}} & \multirow{2}{*}{$\begin{array}{c}\text { Anforderungsniveau } \\
\text { grundlegend }\end{array}$} & \multirow{3}{*}{$\begin{array}{c}\begin{array}{c}\text { Orientierungs- } \\
\text { phase }\end{array} \\
1 \\
1\end{array}$} & \multicolumn{2}{|c|}{ Qualifikationsphase } & \multicolumn{2}{|c|}{\begin{tabular}{l}
\multicolumn{2}{c|}{ Priffung } \\
schr $/$ mdl
\end{tabular}} & \multicolumn{2}{|c|}{\begin{tabular}{|c|c|}
\multicolumn{3}{|c|}{ Gleich mit NatWi } \\
Belegung & Prüftung
\end{tabular}} & \multirow{3}{*}{$\begin{array}{c}\text { Hinweise und Anmerkunger } \\
\text { 1) }\end{array}$} \\
\hline & & & 2 & 2 & $x$ & $\checkmark$ & nein & nein & \\
\hline & erhöht & & $x$ & $\mathrm{x}$ & $x$ & & $x$ & & \\
\hline \multirow{2}{*}{ Bayern } & grundlegend & \multirow{2}{*}{2} & 2 & 2 & $\checkmark$ & $\checkmark$ & nein & ja & 2) \\
\hline & erhöht & & $x$ & $x$ & $x$ & & $x$ & & 3) \\
\hline \multirow{2}{*}{$\begin{array}{l}\text { Berlin } \\
\text { yed } \\
\text { d. }\end{array}$} & grundlegend & \multirow{2}{*}{2} & 3 & 3 & $\checkmark$ & $\checkmark$ & nein & ja & - \\
\hline & erhöht & & 5 & 5 & $\checkmark$ & & nein & & - \\
\hline \multirow{2}{*}{ 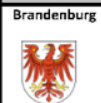 } & grundlegend & \multirow{2}{*}{2} & 3 & 3 & $\checkmark$ & $\checkmark$ & nein & ja & - \\
\hline & erhöht & & 5 & 5 & $\checkmark$ & & nein & & - \\
\hline \multirow{2}{*}{ Bremen } & grundlegend & \multirow{2}{*}{2} & 3 & 3 & $x$ & $\checkmark$ & nein & nein & \multirow{2}{*}{ 4) } \\
\hline & erhöht & & 5 & 5 & $\checkmark$ & & nein & & \\
\hline \multirow{2}{*}{$\begin{array}{l}\text { Hamburg } \\
\text { iditi } \\
\text { idi }\end{array}$} & grundlegend & \multirow{2}{*}{2} & 2 & 2 & $\checkmark$ & $\checkmark$ & nein & ja & 5) \\
\hline & erhöht & & 4 & 4 & $\checkmark$ & & nein & & 6) \\
\hline \multirow{2}{*}{$\begin{array}{l}\text { Hessen } \\
= \\
=\end{array}$} & grundlegend & \multirow{2}{*}{2} & 2 & 2 & $\checkmark$ & $\checkmark$ & nein & ja & \multirow{2}{*}{ 7) } \\
\hline & erhöht & & 5 & 5 & $\checkmark$ & & nein & & \\
\hline \multirow{2}{*}{ 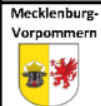 } & grundlegend & \multirow{2}{*}{1} & 3 & 3 & $\checkmark$ & $\checkmark$ & nein & ja & \multirow{2}{*}{ 8) } \\
\hline & erhöht & & 5 & 5 & $\checkmark$ & & nein & & \\
\hline \multirow{4}{*}{\begin{tabular}{|l|} 
Niedersachsen \\
\\
$\begin{array}{l}\text { Nordhein- } \\
\text { Westalen }\end{array}$ \\
3
\end{tabular}} & grundlegend & \multirow{2}{*}{3} & 3 & 3 & $\checkmark$ & $\checkmark$ & ja & ja & 9) \\
\hline & erhöht & & 5 & 5 & $\checkmark$ & & ja & & - \\
\hline & grundlegend & \multirow{2}{*}{2} & 3 & 3 & $\checkmark$ & $\checkmark$ & nein & nein & - \\
\hline & erhöht & & 5 & 5 & $\checkmark$ & & nein & & - \\
\hline \multirow{2}{*}{ Rheinland.Pfala } & grundlegend & 2 & 3 & 3 & $x$ & $\checkmark$ & nein & ja & 10) \\
\hline & erhöht & 4 & 5 & 5 & $\checkmark$ & & nein & & - \\
\hline Saarland & grundlegend & & 2 & 2 & $\checkmark$ & $\checkmark$ & nein & ja & - \\
\hline 3 & erhöht & & 5 & 5 & $\checkmark$ & & nein & & - \\
\hline Sachsen & grundlegend & & 2 & 2 & $x$ & $\checkmark$ & ja & nein & - \\
\hline 륵 & erhöht & & $x$ & $x$ & $x$ & & $x$ & & 11) \\
\hline Sachsen-Anhall & grundlegend & & 2 & 2 & $x$ & $\checkmark$ & nein & nein & - \\
\hline 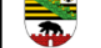 & erhöht & 3 & $x$ & $x$ & $x$ & & $x$ & & - \\
\hline $\begin{array}{l}\text { Schleswig- } \\
\text { Holstein }\end{array}$ & grundlegend & & 3 & 3 & $x$ & $\checkmark$ & nein & nein & - \\
\hline 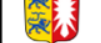 & erhöht & & 4 & 4 & $\checkmark$ & & nein & & 12) \\
\hline Thüringen & grundlegend & & 3 & 3 & $x$ & $\checkmark$ & ja & ja & - \\
\hline 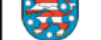 & erhöht & & 5 & 5 & $\checkmark$ & & ja & & 13) \\
\hline
\end{tabular}

verbindlicher Pflichtunterricht für alle SuS

verbindlicher Unterricht nach wahl durch SuS

Wahlunterricht für einige SuS

fachübergreifender Unterricht (Anteil Informatik ausgewiesen) 
sind in den Bundesländern kaum realisiert. Ein Pflichtfach Informatik hat sich bundesweit - nach wie vor - nicht etabliert. Verbindlicher, eigenständiger Informatikunterricht ist sowohl regional als auch auf einzelne Jahrgangsstufen und Schularten beschränkt. Positiv hervorzuheben sind Mecklenburg-Vorpommern, das schulart- und jahrgangsübergreifend ein Pflichtfach „Informatik und Medien“ eingeführt hat, und Sachsen, wo ein eigenständiges Fach Informatik in den Jahrgangsstufen 7-10 für alle Schularten verbindlich ist. Schulartübergreifend gibt es in Baden-Württemberg ein Pflichtfach Informatik für eine Jahrgangsstufe. Dem allgemeinbildenden, weil alle Schularten betreffenden Anspruch, werden Bayern und das Saarland nicht gerecht. Hier gibt es vereinzelt an einigen Schularten bzw. in einigen Jahrgangsstufen verbindlichen Informatikunterricht. In allen anderen Bundesländern wird Informatikunterricht lediglich im Wahlpflicht- oder Wahlbereich angeboten. Bremen und Hessen sind negativ hervorzuheben, da im Sekundarbereich keine Angebote für Informatikunterricht existieren.

Anders ist die Situation, sicher auch historisch bedingt, für das Fach Informatik in der gymnasialen Oberstufe allgemeinbildender Schulen. Informatik kann in allen Bundesländern auf grundlegendem Anforderungsniveau belegt und als Prüfungsfach gewählt werden. In den meisten Fällen ist Informatik als Fach auf erhöhtem Anforderungsniveau wählbar. Im Rahmen einiger länderspezifischer Regelungen ist Informatik hinsichtlich der Belegungsverpflichtungen in der Qualifikationsphase der gymnasialen Oberstufe sowie als Abiturprüfungsfach den naturwissenschaftlichen Fächern gleichgestellt.

Diese Studie zum Informatikunterricht in Deutschland zeigt erneut, dass in den zurückliegenden Jahren viel über Digitalisierung und Medien diskutiert wurde, aber die Bedeutung einer informatischen Bildung für alle Schülerinnen und Schüler und deren Verbindlichkeit offensichtlich nicht erkannt wurde. Es fehlt eine breite Einsicht und Akzeptanz verantwortlicher Stellen sowie ein abgestimmtes und in der Schulpraxis realisierbares Konzept. $\mathrm{Zu}$ diesem Eindruck muss man bei einer Betrachtung der vielgestaltigen hexadezimalen Bildungslandschaft zur informatischen Bildung in Deutschlands Schulen kommen.

Bedenkt man, dass die Veränderungen mit der Industrialisierung vor über 100 Jahren eine Etablierung der Naturwissenschaften in den Schulen zur Folge hatten, könnte dies hinsichtlich des Handelns von heute eine Orientierung geben. Bei den Debatten um Medienbildung, um Datensicherheit und um mögliche Gefahren für Kinder und Jugendliche wird häufig übersehen, dass ein entscheidendes Defizit in der seit Jahren fehlenden Grundlagenbildung zur Informatik - also dem Schulfach Informatik - liegt und damit wichtige Kompetenzen zu den Konzepten der Digitalisierung fehlen. Erst in einem zeitgemäßen Informatikunterricht verstehen die Lernenden die Systeme in ihrer Lebenswelt. Er entmystifiziert Netzwerke, Datenbanken, Verschlüsselung und ebnet den Schülerinnen und Schülern den Weg zum mündigen Informationsbürger. Ein systematischer Fachunterricht legt außerdem die entscheidenden Grundlagen dafür, um in verschiedenen Kontexten konstruktiv die weitere Digitalisierung zu gestalten.

Gleichzeitig erscheint es anhand dieser Studie zur informatischen Bildung in Schulen in Deutschland und mit Blick auf die Entwicklung der Rahmenbedingungen erforderlich, einzelne Aspekte der Digitalisierung in Schulen bildungspolitisch zu diskutieren, um mittelfristig deutliche Veränderungen $\mathrm{zu}$ bewirken.

Mit diesen seit vielen Jahren existierenden Forderungen, gepaart mit konkreten Vorschlägen und dem Nachweis der Realisierbarkeit (in wenigen Bundesländern), stehen wir seitens der Gesellschaft für Informatik nicht allein. Zwei aktuelle Zitate, sowohl seitens der Wissenschaft in den Empfehlungen des Wissenschaftsrates zu den „Perspektiven der Informatik in Deutschland“ [13] als auch seitens der in dem Bereich aktiven gesellschaftlichen Kräfte im Rahmen der „Offensive Digitale Schultransformation“ [14], unterstreichen das erneut und zeigen, dass ein Handeln dringend erforderlich ist.

Der Wissenschaftsrat sieht informatische Bildung als zentralen Schlüssel an, um den digitalen Wandel in der Gesellschaft erfolgreich, inklusiv und nachhaltig zu gestalten. ...

Zwar beobachtet der Wissenschaftsrat einen recht weitgehenden Konsens in Gesellschaft, Politik und Wirtschaft darüber, dass eine Ausweitung der Vermittlung informatischen Wissens und informatischer Kompetenzen in der Schule vonnöten ist - konkret haben beispielsweise immer mehr Landesregierungen zuletzt beschlossen, in kleinen Schritten zukünftig verpflichtenden Informatik-Unterricht einzuführen. Zweifel sind angebracht, ob die Geschwindigkeit dieser positiven Entwicklung ausreicht angesichts der Tatsache, dass Deutschland noch weit entfernt ist von einer flächendeckenden und kontinuierlichen Vermittlung informatischer Bildung an alle Schülerinnen und Schüler. Auch im europäischen Vergleich besteht Nachholbedarf.

Neben dem übergeordneten Ziel einer digitalen Mündigkeit aller Bürgerinnen und Bürger hält der Wissenschaftsrat schulische informatische Bildung aber auch vor dem Hintergrund der Notwendigkeit, mehr Absolventinnen und Absolventen von Informatik-Studiengängen hervorzubringen, für dringend erforderlich: Über Informatik-Unterricht in der Schule können bei Schülerinnen und Schülern frühzeitig Begeisterung für das Fach geweckt und ein diverserer Kreis an In- 
teressierten für ein Studium gewonnen werden. Auch kann die schulische Bildung einen Beitrag zur Senkung der Studienabbruchsquoten im Studienbereich Informatik leisten, indem frühzeitig Grundkenntnisse und realistische Vorstellungen von einem InformatikStudium vermittelt werden. Der Wissenschaftsrat ermutigt die Länder, die schnelle und flächendeckende Einführung informatischer Bildung in den Schulen noch stärker zu priorisieren, als dies bisher vorgesehen ist [13, S. 72].

\section{Verpflichtenden Informatikunterricht ausweiten und die Nutzung von digitalen Werkzeugen in al- len Fächern verbessern}

Alle Kinder und Jugendliche, die auf IT-Systeme im schulischen und außerschulischen Kontext angewiesen sind, benötigen informatische Grundlagen, um anwendungsbezogene, technische und gesellschaftliche Perspektiven digitaler Technologien einschätzen zu können. Dazu sollte bundesweit flächendeckender Informatikunterricht ab der Sekundarstufe I angeboten werden, der auf alle drei Perspektiven eingeht und so die Medienbildung ergänzt und unterstützt [14].

Funding Open Access funding enabled and organized by Projekt DEAL.

Open Access Dieser Artikel wird unter der Creative Commons Namensnennung 4.0 International Lizenz veröffentlicht, welche die Nutzung, Vervielfältigung, Bearbeitung, Verbreitung und Wiedergabe in jeglichem Medium und Format erlaubt, sofern Sie den/die ursprünglichen Autor(en) und die Quelle ordnungsgemäß nennen, einen Link zur Creative Commons Lizenz beifügen und angeben, ob Änderungen vorgenommen wurden.

Die in diesem Artikel enthaltenen Bilder und sonstiges Drittmaterial unterliegen ebenfalls der genannten Creative Commons Lizenz, sofern sich aus der Abbildungslegende nichts anderes ergibt. Sofern das betreffende Material nicht unter der genannten Creative Commons Lizenz steht und die betreffende Handlung nicht nach gesetzlichen Vorschriften erlaubt ist, ist für die oben aufgeführten Weiterverwendungen des Materials die Einwilligung des jeweiligen Rechteinhabers einzuholen.

Weitere Details zur Lizenz entnehmen Sie bitte der Lizenzinformation auf http://creativecommons.org/licenses/by/4.0/deed.de.

\section{Literatur}

1. KMK (2016) Kultusministerkonferenz: „Bildung in der digitalen Welt" Beschluss vom 8. Dez. 2016 in der Fassung vom 7. Dez. 2017. Verfügbar unter: https://www.kmk.org/themen/bildungin-der-digitalen-welt/strategie-bildung-in-der-digitalen-welt.html. Zugegriffen: 25. März 2021

2. Hellmig L (2019) Digitalisierung an Schulen: Informatik für alle Eine Analyse von Julia Bernewasser. Zeit-Online vom 9. Mai 2019. Verfügbar unter: https://www.zeit.de/gesellschaft/schule/201905/digitalisierung-schulen-informatik-unterricht-programmierendigitalpakt. Zugegriffen: 25.März 2021

3. Friedrich S (2017) Bildung in der digitalen Welt - Anmerkungen zum Strategiepapier der KMK. LOG IN 187/188:10-17

4. Gesellschaft für Informatik (2008) Grundsätze und Standards für die Informatik in der Schule - Bildungsstandards Informatik für die Sekundarstufe I. Empfehlungen der Gesellschaft für Informatik e.V. Beilage zu LOG IN, 28(150/151). https://www. informatikstandards.de. Zugegriffen: 25. März 2021

5. Gesellschaft für Informatik (2016) Bildungsstandards Informatik für die Sekundarstufe II - Empfehlungen der Gesellschaft für Informatik e.V. Beilage zu LOG IN, 36(183/184). https://www. informatikstandards.de. Zugegriffen: 25. März 2021

6. Gesellschaft für Informatik (2019) Kompetenzen für informatische Bildung im Primarbereich - Empfehlungen der Gesellschaft für Informatik e.V. Beilage zu LOG IN 39.(191/192). https://www. informatikstandards.de. Zugegriffen: 25. März 2021

7. Giesecke H (1999) Vom Sinn der Bildung. Funkmanuskripte 7. http://www.hermann-giesecke.de/Funk7.pdf. Zugegriffen: 25. März 2021

8. Schulen an das Netz (1995) Bildungsinitiative Informatik und Telekommunikation der Gesellschaft für Informatik e. V., Bonn (Sonderdruck). https://gi.de/themen/beitrag/macht-denselben-fehlernicht-ein-zweites-mal. Zugegriffen: 25. März 2021

9. Starruß I (2010) Informatikunterricht in Deutschland. Analyse der informatischen Bildung an allgemeinbildenden Schulen auf der Basis der im Jahr 2010 gültigen Lehrpläne. Dresden. https://docplayer. org/50806106-Synopse-zum-informatikunterricht-in-deutschland. html. Zugegriffen: 25. März 2021

10. Vollstädt W et al (1999) Lehrpläne im Schulalltag: Eine empirische Studie zur Akzeptanz und Wirkung von Lehrplänen in der Sekundarstufe I. VS, Wiesbaden

11. Schwarz R (2020) Informatikunterricht in Deutschland. Darstellung der Situation in den 16 Bundesländern im Jahr 2020. Rostock. https://pidi.informatik.uni-rostock.de/lehre/abschlussarbeiten/haus arbeiten/. Zugegriffen: 25. März 2021

12. Gesellschaft für Informatik (2021) Informatik-Monitor. https:// informatik-monitor.de. Zugegriffen: 25. März 2021

13. Wissenschaftsrat (2020) Perspektiven der Informatik in Deutschland. https://www.wissenschaftsrat.de/download/2020/8675-20. pdf. Zugegriffen: 25. März 2021

14. Offensive Digitale Schultransformation (2020) GI Berlin. https:// offensive-digitale-schultransforma

tion.de/. Zugegriffen: 25. März 2021 